\title{
Wind reconstruction algorithm for Viking Lander 1
}

\author{
Tuomas Kynkäänniemi ${ }^{1}$, Osku Kemppinen ${ }^{2, a}$, Ari-Matti Harri ${ }^{2}$, and Walter Schmidt ${ }^{2}$ \\ ${ }^{1}$ School of Science, Aalto University, Espoo, Finland \\ ${ }^{2}$ Earth Observation, Finnish Meteorological Institute, Helsinki, Finland \\ ${ }^{a}$ currently at: Department of Physics, Kansas State University, Manhattan, Kansas, USA
}

Correspondence to: Tuomas Kynkäänniemi (tuomas.kynkaanniemi@fmi.fi)

Received: 8 December 2016 - Discussion started: 22 February 2017

Revised: 18 May 2017 - Accepted: 19 May 2017 - Published: 22 June 2017

\begin{abstract}
The wind measurement sensors of Viking Lander 1 (VL1) were only fully operational for the first 45 sols of the mission. We have developed an algorithm for reconstructing the wind measurement data after the wind measurement sensor failures. The algorithm for wind reconstruction enables the processing of wind data during the complete VL1 mission. The heater element of the quadrant sensor, which provided auxiliary measurement for wind direction, failed during the 45th sol of the VL1 mission. Additionally, one of the wind sensors of VL1 broke down during sol 378. Regardless of the failures, it was still possible to reconstruct the wind measurement data, because the failed components of the sensors did not prevent the determination of the wind direction and speed, as some of the components of the wind measurement setup remained intact for the complete mission.

This article concentrates on presenting the wind reconstruction algorithm and methods for validating the operation of the algorithm. The algorithm enables the reconstruction of wind measurements for the complete VL1 mission. The amount of available sols is extended from 350 to 2245 sols.
\end{abstract}

\section{Introduction}

The primary goal of the Viking mission was to investigate the current or past existence of life on Mars. The Viking Lander's payload (Soffen, 1977) consisted of instruments designed for meteorological experiments (Chamberlain et al., 1976), for seismological measurements and for experiments on the composition of the atmosphere.

Viking Lander 1 (VL1) landed on Mars on 20 July 1976. The location of the landing spot was a low plain area named
Chryse Planitia, which has a slope rising from south to west. The landing coordinates of VL1 were $22^{\circ} \mathrm{N}, 48^{\circ} \mathrm{W}$.

VL1 operated for 2245 Martian sols, which is much longer than was expected (Soffen, 1977). Thus the data set produced is significant in size. The VL1 data set enables the study of various meteorological phenomena ranging from diurnal to seasonal timescales using measurements of temperature, pressure and wind direction.

The Finnish Meteorological Institute (FMI) has developed a set of tools that enable processing the Viking Lander meteorological data beyond previously publicly available data in the National Aeronautics and Space Administration's (NASA) Planetary Data System (PDS; Kemppinen et al., 2013). Currently NASA's PDS contains wind measurement data only from 350 sols. The FMI tools make it possible to process data from the full 2245 sols instead, over a sixfold increase.

The state of the wind measurements from the surface of Mars is quite limited, because the wind measurements of other missions to the surface of Mars have either failed or produced a shorter data set than that of VL1. After the Viking mission there was a 20-year gap before the research on the surface of Mars was finally resumed. The Mars Pathfinder (MPF) conducted wind measurements on the surface of Mars for 86 sols (Golombek et al., 1999), the results of which are presented in Schofield et al. (1997). The MPF data are not available in NASA's PDS. The Phoenix Lander performed wind speeds and directions measurements with a mechanical anemometer from a polar region of Mars $\left(68.22^{\circ} \mathrm{N}\right.$, $\left.125.75^{\circ} \mathrm{W}\right)$ (Holstein-Rathlou et al., 2010). The length of the Phoenix mission was 152 sols. The Mars Science Laboratory (MSL) could surpass the extent of the VL1 data set in the year 2018, but the wind measurement setup of the MSL is 
not fully functional due to hardware issues. It is still possible to use the MSL wind sensor to retrieve wind speeds and accurate wind directions if the wind comes from the hemisphere in front of the rover (Newman et al., 2016). Thus, the VL1 wind measurement data set remains the longest data set of wind measurements from the surface of Mars at least for the coming years.

This article focuses on the reconstruction of the VL1 wind measurements. Section 2 provides an overview of the VL1 wind sensors and their malfunctions. The wind reconstruction algorithm is defined in Sect. 3, and the validation methods for the algorithm are presented in Sect. 4. The reconstructed data from the VL1 wind measurements are presented in Sect. 5.

The significance of this work lies in the fact that other missions on the surface of Mars have not yet succeeded in measuring a data set equal to the size of that of VL1. Even though the VL1 landed on Mars 40 years ago, not all the data from the VL1 wind instruments have been analyzed and published due to various complications that are described below.

\section{Wind measurement setup and sensor malfunctions}

\subsection{Wind sensors}

The Viking Lander wind measurement setup consisted of two hot-film wind sensors and a quadrant sensor (Chamberlain et al., 1976). The hot-film wind sensors were designed to determine the wind velocity component normal to each of the sensors, and the quadrant sensor was designed to provide information about the wind direction and therefore solve the fourfold ambiguity of the normal components (Chamberlain et al., 1976).

The wind sensor design is presented in Fig. 1. The hotfilm wind sensors were mounted at a $90^{\circ}$ angle with respect to each other, and the temperature of the films was maintained at $100^{\circ} \mathrm{C}$ above the ambient gas temperature. The sensor convective heat transfer can be represented by the Nusselt number:

$N u=\frac{Q}{\pi L \kappa_{F} \Delta T}$,

where $Q$ is the power convected to the fluid, $L$ is the element length, $\kappa_{F}$ is the gas thermal conductivity (at the gas film temperature) and $\Delta T$ is the element overheating temperature. The wind velocity normal to each hot-film sensor could be determined from the power required to maintain the overheating temperature against heat loss due to radiation and conduction. Assuming the wind velocity is a vector $\boldsymbol{v}$ from direction $\theta$, as presented in Fig. 1, the perpendicular wind speed components $v_{x}$ and $v_{y}$ can be determined by

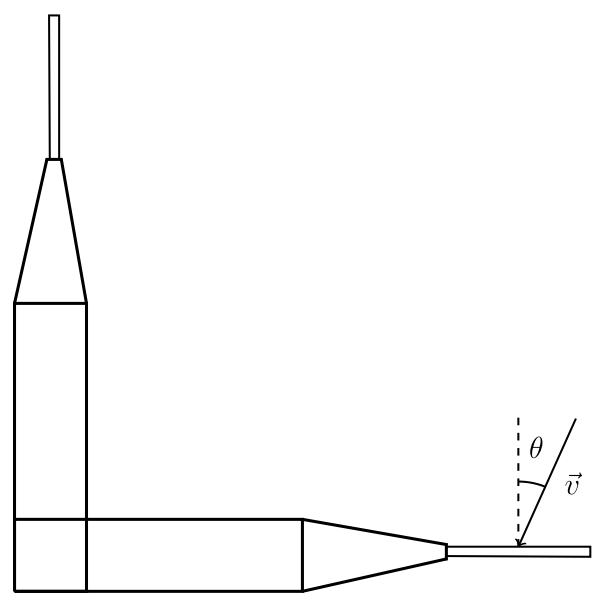

Figure 1. Wind sensor design. The hot films were mounted at the end of two separate holders, which were set perpendicular to each other (Davey et al., 1973).

$v_{y}=|\boldsymbol{v}| \sin (\theta)$,

$v_{x}=|\boldsymbol{v}| \cos (\theta)$.

There exists a fourfold ambiguity in the wind velocity measured by the two wind sensors. The ambiguity is caused by the wind sensors only measuring the wind velocity component normal to the sensor, and it is resolved using the quadrant sensor (Davey et al., 1973; Sutton et al., 1978).

\subsection{Quadrant sensor}

The quadrant sensor was designed to provide a secondary measurement to solve the ambiguity in the wind direction. The design of the sensor is presented in Fig. 2. The operating principle of the sensor is based on locating the thermal wake of a heated vertical cylinder. The location of the wake is determined from the temperature distribution about the cylinder using four chromel-constantan thermocouples (TCs). These thermocouples were connected in series, and each pair measures the temperature difference across the sensor due to the thermal wake (Davey et al., 1973; Sutton et al., 1978).

\subsection{SANMET}

The data used in the wind reconstruction algorithm are from NASA's Science Analysis of Meteorology (SANMET) program (Buehler, 1974). SANMET calculates the values of various meteorological quantities - such as wind speed, wind direction, temperature and pressure - from the voltage signals of the meteorology instruments. The operating principle and methods used by the SANMET program are presented in detail in Buehler (1974). The wind reconstruction algorithm requires the voltages $V_{\mathrm{QS}_{1}}$ and $V_{\mathrm{QS}_{2}}$ of the quadrant sensor's thermocouples. These voltages can be read from the 


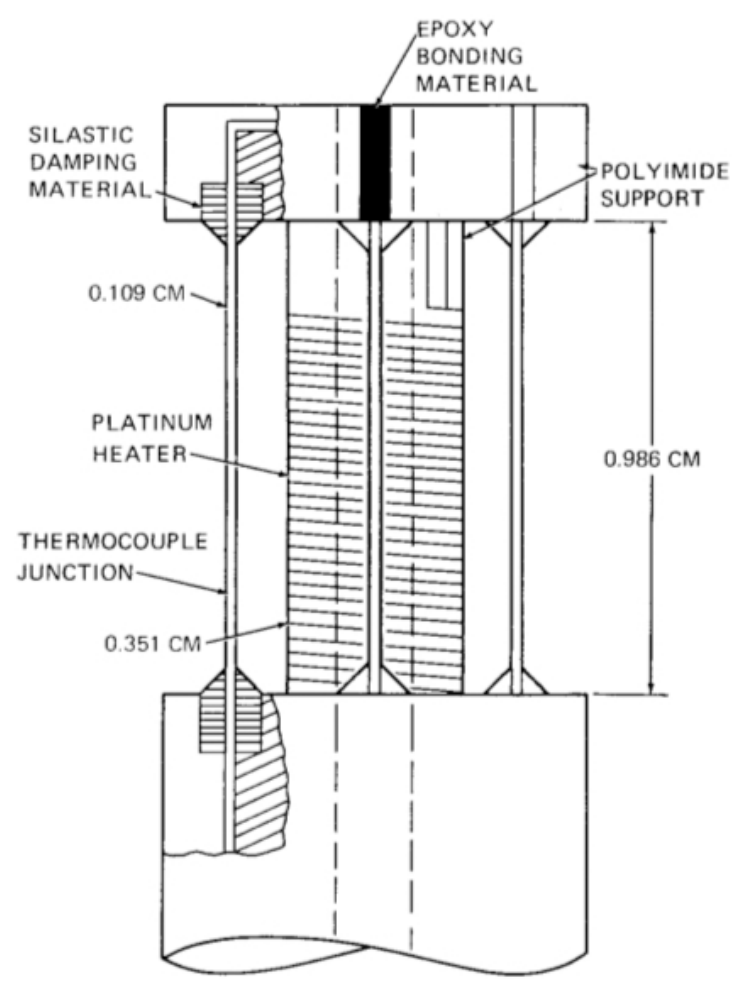

Figure 2. Quadrant sensor design (Hess et al., 1977).

SANMET output under the DATA6 header. The algorithm additionally requires the wind directions $\theta$ and the Nusselt numbers $N u_{1}$ and $N u_{2}$ of the wind sensors during sols 1-45.

\subsection{VL1 sensor malfunctions}

The heater element of the VL1 quadrant sensor was thought to be damaged during the 45th sol (Murphy et al., 1990; Hess et al., 1977). During sol 46 there exists a sudden change in the behavior of the voltage values of the thermocouple pairs QS1 and QS2, which is shown in Fig. 3. When the quadrant sensor was functioning in the intended way, the voltage values of QS1 and QS2 varied within $\pm 5.0 \mathrm{mV}$, but after the failure of the heater element the variation range of the voltages changed to approximately $\pm 0.5 \mathrm{mV}$.

After the failure of the quadrant sensor's heater element, the SANMET process for determining the wind direction was no longer fully reliable. It is assumed that both of the quadrant sensor thermocouples remained functional for the whole VL1 mission. Therefore the instrument can be used whenever the radiance of the Sun is strong enough to heat the quadrant sensor's heater element to a temperature where the bias-corrected thermocouples' signals exceeded $0.05 \mathrm{mV}$.

In addition to the failure of the quadrant sensor's heater element, one of the two hot-film wind sensors of VL1 broke down during sols 377-378. The decay of the VL1 wind sensor 2 is illustrated in Fig. 4 by presenting the unbinned values of the Nusselt number measured by both of the wind sensors.

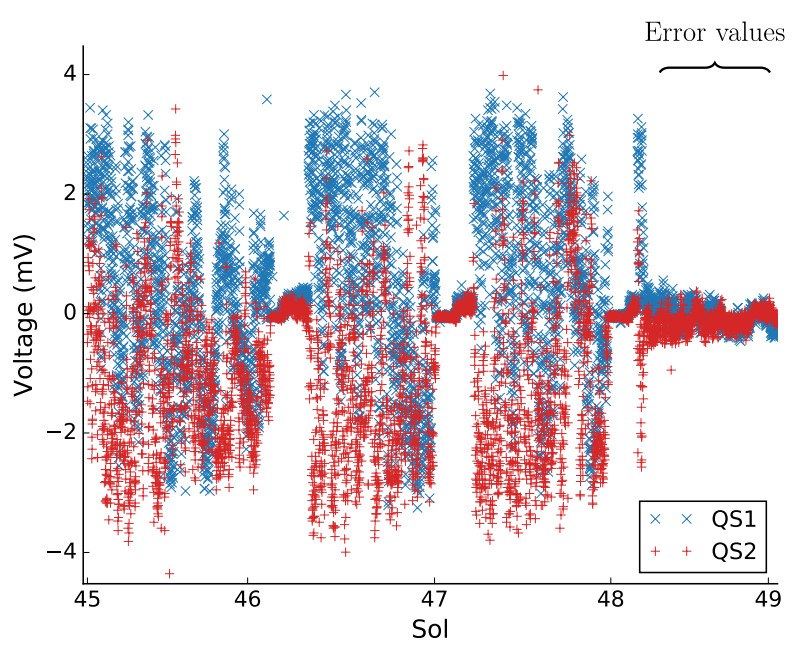

Figure 3. Failure of the heater element of VL1's quadrant sensor during sol 46.

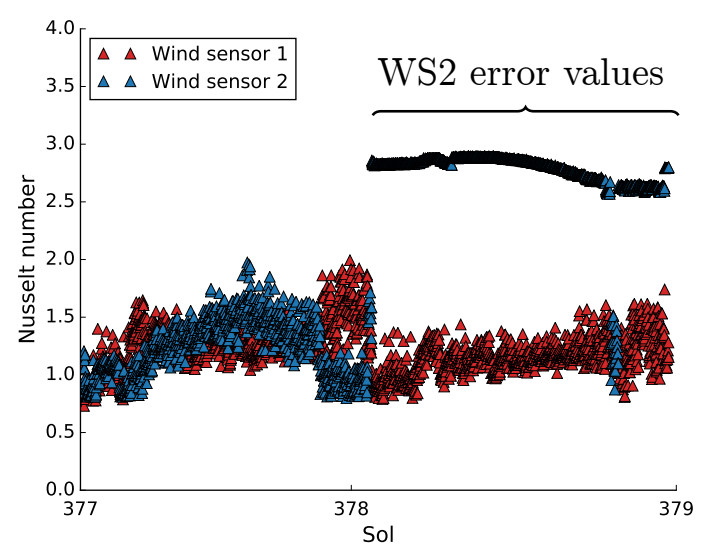

Figure 4. The decay of the VL1's wind sensor 2 (WS2) during sols 377-378.

At the turn of sols 377 and 378, the dynamic of the wind sensor 2 changed so that the sensor obtains almost constant Nusselt number of slightly less than 3.0. At the end of sol 378's measurements the wind sensor 2 resumed nominal function for a very short period of time, after which the behavior of the sensor returned to the failure state permanently.

\section{Wind reconstruction algorithm}

\subsection{The reanalyzing process of the wind measurements}

Due to the failures of the quadrant sensor and one of the hotfilm wind sensors, the reconstruction of wind directions and speeds have to be carried out in two stages. The complete workflow of the wind reconstruction algorithm is presented in the schema of Fig. 5. Both of the stages first contain a calibration phase, where the calibration function is calculated, 


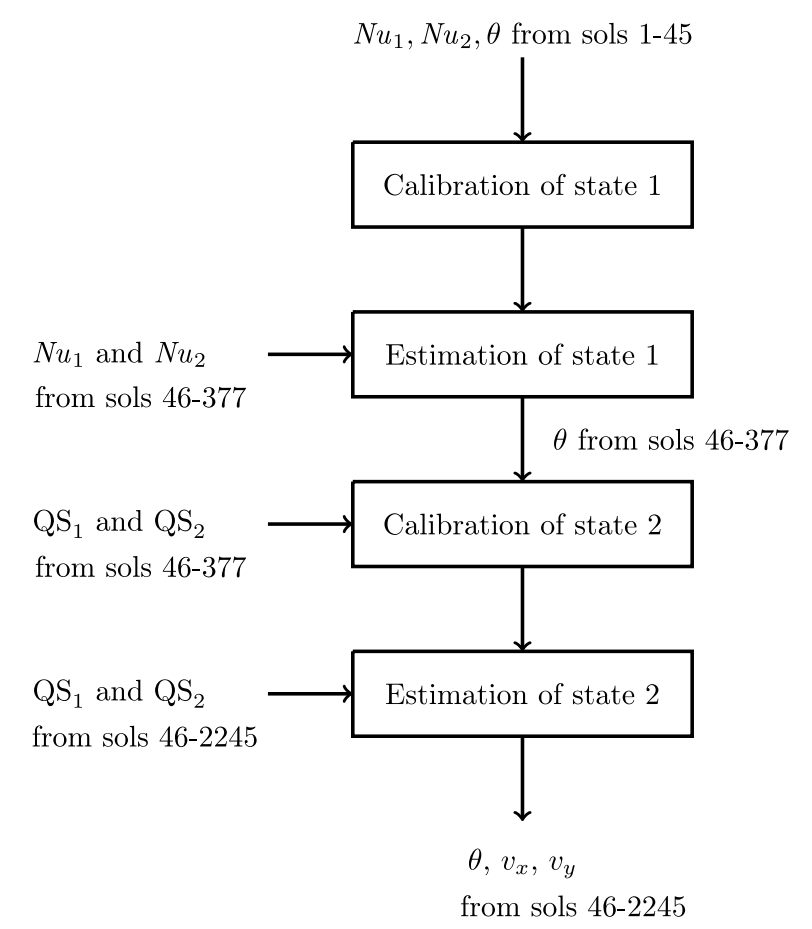

Figure 5. Schema of the complete processing chain of the wind reconstruction algorithm. $N u_{1}$ and $N u_{2}$ are the Nusselt numbers of wind sensors 1 and $2, \mathrm{QS}_{1}$ and $\mathrm{QS}_{2}$ are the voltage values of the thermocouple pairs, $\theta$ is the wind direction, and $v_{x}$ and $v_{y}$ are the wind speed normal components.

and then an estimation phase, where the wind direction $\theta$ is estimated.

In the first stage the wind directions of the fully functional quadrant sensor are used to calibrate the two hot-film sensors' Nusselt numbers. The method of calibrating the Nusselt numbers with the correct wind direction data from sols 1-45 is presented in Sect. 3.2. The Nusselt numbers of the two hotfilm wind sensors are then used for determining the wind direction during sols $46-377$. The reconstruction of wind direction after sol 376 is unfortunately impossible using the calibrated Nusselt numbers, as one of the two sensors failed during sol 378. Therefore in the second stage, the reconstructed wind directions from sols 46-377 are required for calibrating the weak voltage signals of the quadrant sensor, to enable the estimation of wind direction after sol 377 with the quadrant sensor.

In the second stage the reconstructed wind directions, determined using the Nusselt numbers of wind sensors, are used to calibrate the quadrant sensor voltages. The quadrant sensor thermocouple pairs continued working nominally after the heater element failure. The voltages observed by the thermocouples are weaker and contain more noise, but they have the same dynamic as during sols $1-45$, when the heater element was intact. Section 3.5 presents a method for calibrat- ing the quadrant sensor voltages with the reconstructed wind directions from sols 46-377.

After calibrating the voltages of the quadrant sensor, the wind directions can be solved for sol 377 and beyond. With the reconstructed wind direction and one stream of intact wind component data the wind speeds can be solved for sol 377 and onwards. The complete VL1 mission was reconstructed using the calibrated quadrant sensor signals, which were calibrated using the Nusselt number wind direction estimates from stage one.

\subsection{Calibration of the wind sensors}

The wind direction can be obtained from the Nusselt numbers. This is because the Nusselt numbers are dependent on the wind components normal to the hot-film sensors. The first stage of the wind reconstruction algorithm relies on the assumption that the wind sensors get similar values for Nusselt numbers during the period of interest to those during sols $1-45$. The values of Nusselt numbers for both wind sensors were examined from arbitrary sols from the sol interval 46377 of the VL1 mission. The results from this spot check were encouraging as the Nusselt numbers of the wind sensors remained the same order of magnitude during the examined sols. The method of using the Nusselt numbers for estimating the wind direction was first studied by Murphy et al. (1990). The method used here is similar to method used in Murphy et al. (1990), but it contains less subjectivity in the process as the wind directions are not reconstructed mechanically by hand.

The reconstruction algorithm begins by computing first the calibration functions $F_{i}$, for different wind velocity classes, from the Nusselt numbers $N u_{1}$ and $N u_{2}$ measured by the wind sensors. The calibration functions $F_{i}$ are defined the same way as in Murphy et al. (1990):

$F=\frac{\left(N u_{1}-N u_{2}\right)}{\sqrt{N u_{1} N u_{2}}}$.

After the values for $F$ were calculated by Eq. (4), they were binned into $1^{\circ}$-sized bins. Then, 12th-order polynomials were fitted to the binned values calculated with Eq. (4). The polynomials with degree $n=1, \ldots, 15$ were considered. For 12th-order polynomials, the mean absolute difference of the reconstructed angle and the SANMET-provided "correct" angle from sols $1-45$ was the smallest.

Because the Nusselt numbers depend on the wind velocity, the velocities were divided into four different velocity classes. For each velocity class the calibration function was obtained using the method described earlier. Table 1 presents the different velocity classes for which the calibration functions $F_{i}$ were determined. Wind velocities greater than $50 \mathrm{~ms}^{-1}$ were quite rare during the first 45 sols of VL1. Thus, there were not enough data of the Nusselt numbers 


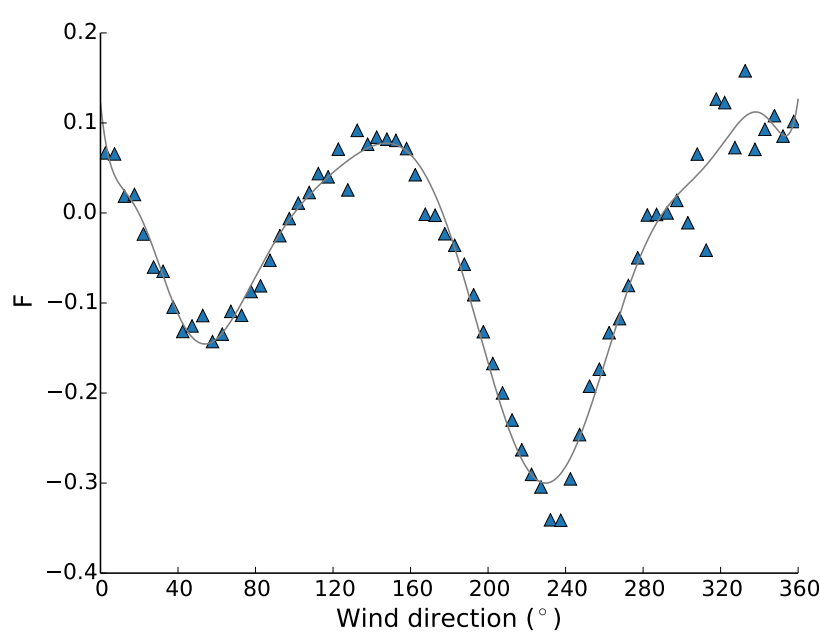

Figure 6. Calibration function $F_{1}$ for velocity class 1 .

Table 1. The velocity classes of the wind reconstruction algorithm.

\begin{tabular}{lr}
\hline Class number & Wind velocity \\
\hline 1 & $<2 \mathrm{~ms}^{-1}$ \\
2 & $2-4 \mathrm{~ms}^{-1}$ \\
3 & $4-8 \mathrm{~ms}^{-1}$ \\
4 & $8-50 \mathrm{~ms}^{-1}$ \\
\hline
\end{tabular}

from these velocities to produce good statistics, and the velocities were ignored.

The calibration functions $F_{i}$ for each wind class are plotted in Figs. 6, 7, 8 and 9 with gray-colored curves. Although there exists a slight overfitting effect in the calibration functions in Figs. 6, 8 and 7, the calibration functions can distinguish the wind directions from different $\mathrm{F}$ values as the data are distributed uniformly to the full angle interval. For the calibration in Fig. 9 the data are not distributed evenly to the complete angle interval, which reduces the accuracy of the wind direction estimates.

The data for the calibration functions in Figs. 6, 8, 7 and 9 were obtained from the first 45 sols, when the quadrant sensor was still fully functional, and it consisted of 53229 measurement samples. Most of the samples were from conditions where the wind velocity was less than $8.0 \mathrm{~ms}^{-1}$.

To reconstruct the wind directions of a complete sol, the algorithm calculates for each measurement sample the value of function $F$ using Eq. (4) and determines the velocity class of the sample. With the calculated $F$ value, the roots $\theta_{i}$ of 12th-order polynomial were calculated by solving

$\sum_{i=0}^{12} a_{i} \theta^{i}-F_{\text {sample }}=0$.

The coefficients $a_{i}$ of the polynomial were obtained from the calibration function fit. The roots $\theta_{i}$ of Eq. (5) were used

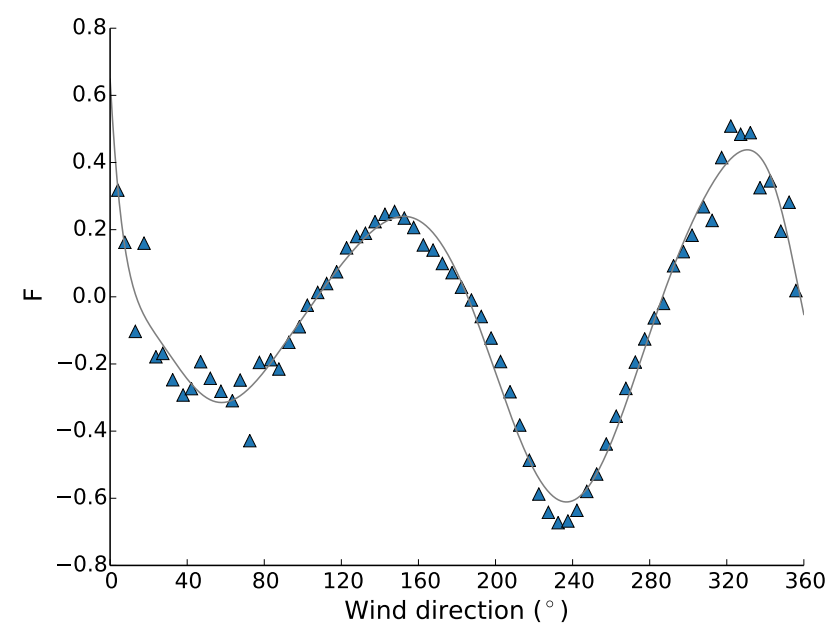

Figure 7. Calibration function $F_{3}$ for velocity class 3 .

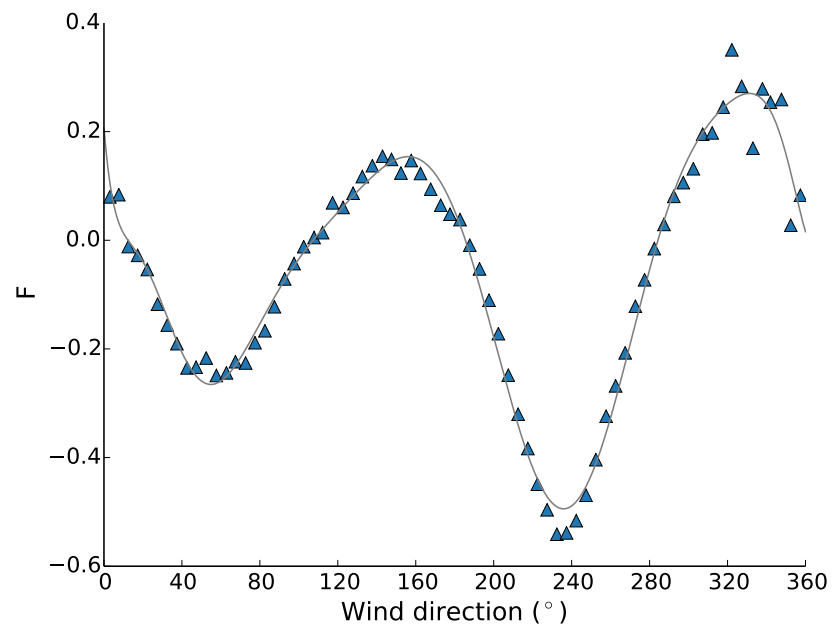

Figure 8. Calibration function $F_{2}$ for velocity class 2 .

as candidate angles for wind direction. This set of angles usually contains either twofold or fourfold ambiguity, which is resolved using either the quadrant sensor signals or time continuity. It is possible that for a certain sample there are no roots for Eq. (5) of the calibration functions; therefore the candidate angles for wind direction can not be solved. In this situation an error value is placed as the value of the particular sample.

When the absolute value of the bias-corrected quadrant sensor voltages exceeded the threshold value $(0.05 \mathrm{mV})$, the correct quadrant for wind direction was established from the quadrant sensor signals using a look-up table presented in Table 2. The look-up table is based on the intended operating principle of the quadrant sensor and the orientation of the VL1 wind measurement instruments, which is shown in Fig. 10. The quadrant sensor thermocouple pair $\mathrm{QS}_{1}$ has positive voltage values when the wind is from TC-1 to TC-2; the 


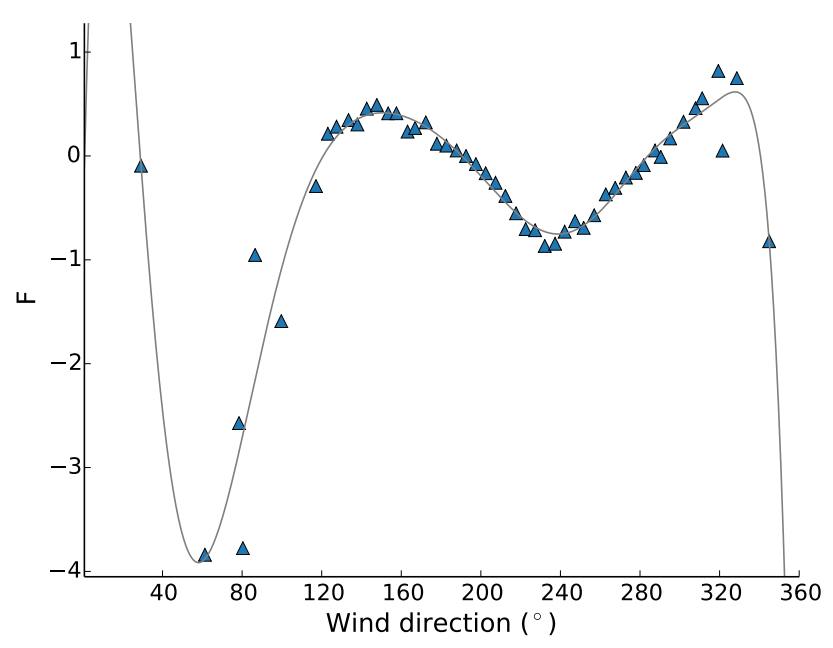

Figure 9. Calibration function $F_{4}$ for velocity class 4 .

Table 2. VL1's look-up table of the developed wind reconstruction algorithm.

\begin{tabular}{lrr}
\hline Angle range & $V_{\mathrm{QS}_{1}}$ & $V_{\mathrm{QS}_{2}}$ \\
\hline $350-80^{\circ}$ & $<0$ & $>0$ \\
$80-170^{\circ}$ & $<0$ & $<0$ \\
$170-260^{\circ}$ & $>0$ & $<0$ \\
$260-350^{\circ}$ & $>0$ & $>0$ \\
\hline
\end{tabular}

thermocouple pair $\mathrm{QS}_{2}$ has positive voltage values when the wind is from TC-3 to TC-4.

The ambiguity of the wind direction is settled by selecting from the set of candidate angles the specific angle that is in the correct wind quadrant, as determined by the quadrant sensor voltages. If there are many candidate angles in the same wind quadrant, the wind direction is arbitrarily selected to be the smallest angle from the set of candidate angles.

For cases of the quadrant sensor voltages not exceeding the threshold value, time continuity is used for determining the wind direction. Time continuity works as follows: the chosen candidate angle is the one nearest to the last-determined angle, albeit only if the time difference between that one and the current one is less than $1 \mathrm{~h}$. This principle is sufficient when the elapsed time between the measurement samples is not too long, as wind often exhibits continuous behavior. However, if the time between the two samples exceeds the threshold of $1 \mathrm{~h}$, the use of time continuity is likely not valid, and in these cases the dynamic wind table (DWT) is used to determine the correct angle. The operation principle of the DWT is described in Sect. 3.3. The operation of the first stage in the wind reconstruction algorithm is summarized in Algorithm 1 .

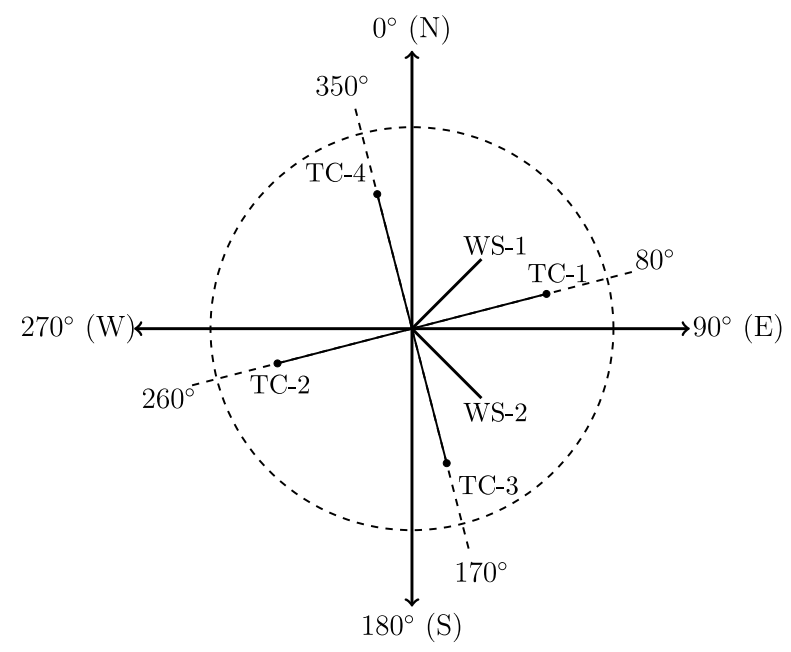

Figure 10. The orientation of VL1's wind sensor assembly, adapted from Murphy et al. (1990). The degree coordinate axis refers to the Martian planetary directions. The abbreviations TC and WS stand for thermocouple and wind sensor, respectively.

\subsection{Dynamic wind table}

Time continuity was used for approximately $60 \%$ of the reconstructed angles of VL1 during sols 46-377. The DWT was developed to determine the wind direction when the use of time continuity was not possible. Notably, the DWT is capable of taking into account Mars' seasonal variations in wind direction.

The DWT was implemented using a hash table, with the Lander Local Time (LLT) in hours as the key. The value of the table is a queue containing the mean value of the reconstructed angles for the corresponding hour. When reconstructing a new sol, new values for hourly mean wind direction were calculated and then added to the DWT's queue. The DWT's queue for hourly mean wind direction will hold data at most from the last 10 reconstructed sols.

When the use of time continuity was required due to the quadrant sensor voltages being too low, the time difference in hours between the current sample and last-measured sample was solved. In the case of the time difference of samples being more than $1 \mathrm{~h}$, the time value was used to obtain the mean wind direction of the hour from those of the last 10 sols where data from the hour in question were recorded. A maximum allowed rate of wind direction change was defined to prevent error values filling the DWT. If the allowed rate of change is exceeded, the wind direction is not added to the DWT, thus preventing outliers from distorting the correct average values.

\subsection{Bias correction of the quadrant sensor voltages}

The bias corrections of the thermocouple pairs QS1 and QS2 do not stay constant during the VL1 mission. There exists a 


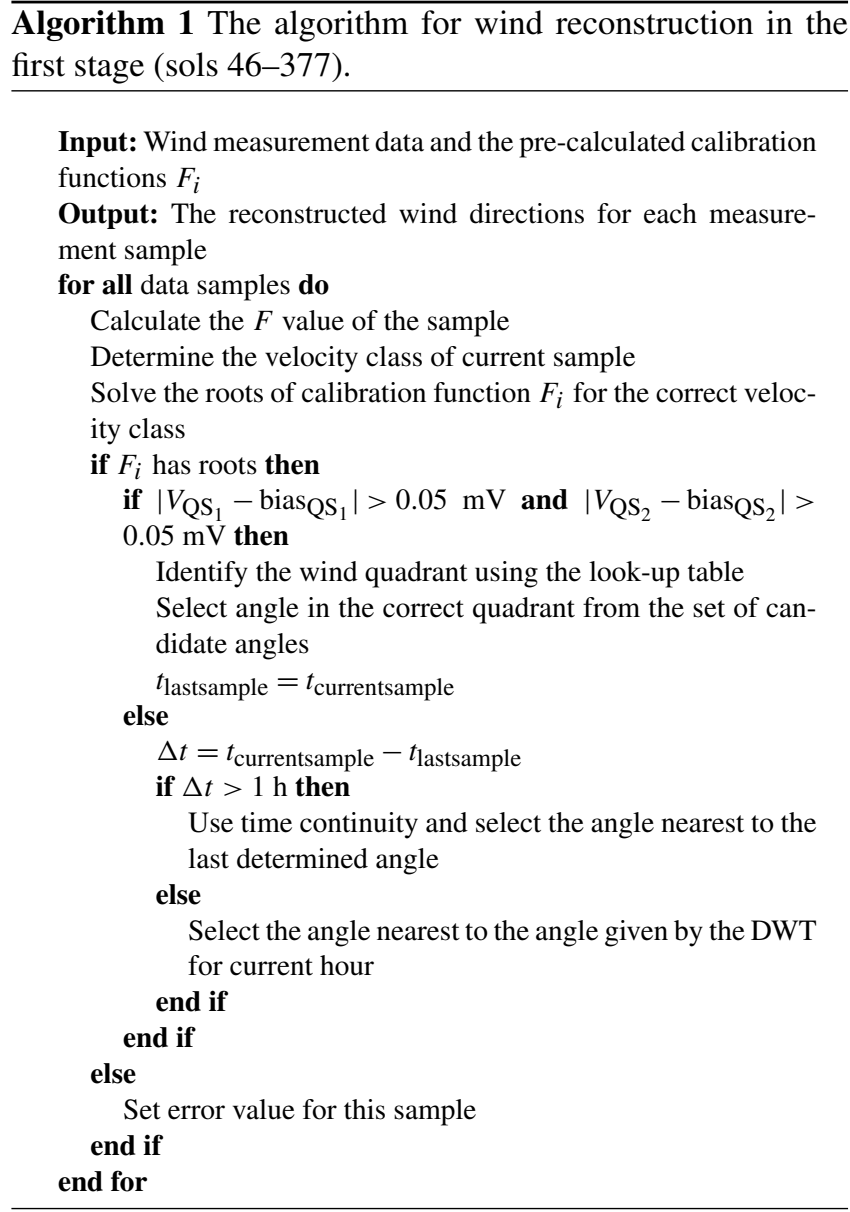

drift in both of the pairs' voltages. Therefore a diurnal bias correction for QS1 and QS2 is required to distinguish more reliably the correct quadrant sensor signals from noise. The samples used in determining the bias corrections were from nighttime records during light-wind conditions.

The criterion for nighttime was defined to be that the sample is measured between 00:00 and 06:00 LLT, and the criterion for light-wind conditions was that the wind velocity is less than $0.8 \mathrm{~ms}^{-1}$. The voltage values of the samples which meet these two criteria were filtered from all the measured samples, and the diurnal mean values of voltages were calculated. A linear fit was made to the calculated mean voltages to obtain the diurnal bias correction for those sols where the wind direction is reconstructed. The diurnal mean voltage values of the thermocouple pairs and linear fit are presented in Figs. 11 and 12. Even though the thermocouple pairs QS1 and QS2 are almost identical, the bias voltage of QS1 changed more during sols 46-377 than QS2 bias voltage.

\subsection{Calibration of the quadrant sensor}

In the second stage of the wind reconstruction algorithm, the recalibration of the quadrant sensor signals is required. For

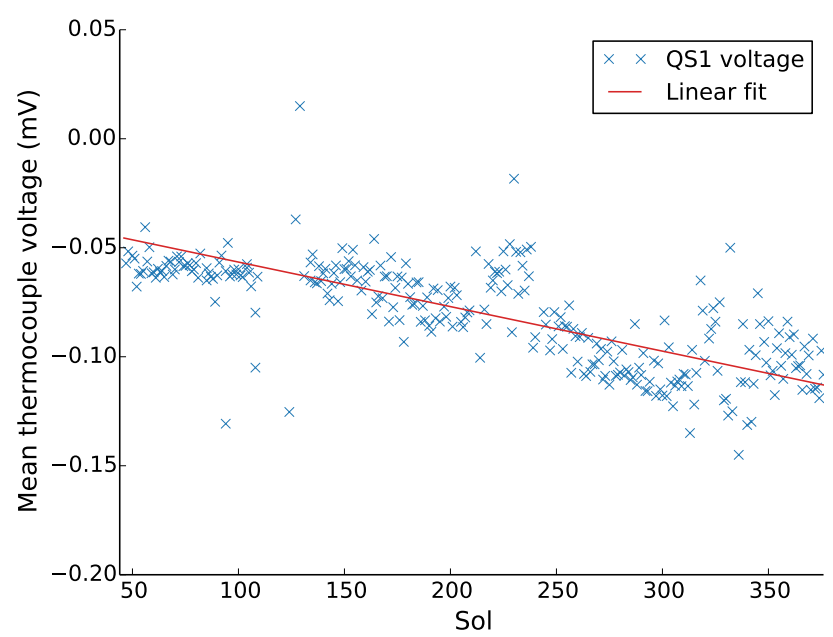

Figure 11. Bias correction of QS1.

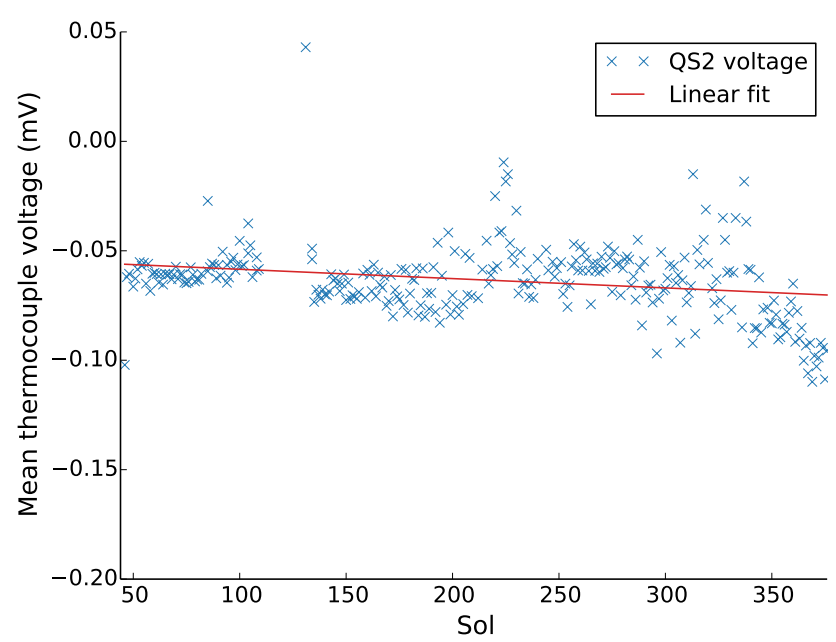

Figure 12. Bias correction of QS2.

calibrating the voltages of the quadrant sensor, a second calibration function, called $R$, was defined as

$R=\frac{V_{\mathrm{QS}_{1}}}{V_{\mathrm{QS}_{2}}}$,

which is the ratio of the thermocouple voltages $V_{\mathrm{QS}_{1}}$ and $V_{\mathrm{QS}_{2}}$. When the calibration function $R$ is plotted as a function of wind direction, a tangential function is observed, as shown in Fig. 13. The tangential function arises from the geometry of the quadrant sensor. There exist angles $180^{\circ}$ apart for both thermocouples where the wind velocity vector is perpendicular to the thermocouple pair QS2. In this case, the temperature difference, measured by the thermocouple, is zero, and the thermocouple can not detect the wind flow from this direction. To fit a tangential function into the $R$ data of the quadrant sensor, the following model was used: 
Table 3. The values of parameters $A, B$ and $C$ obtained from the LSQ fits.

\begin{tabular}{lrrr}
\hline Sol interval & $A$ & $B$ & $C$ \\
\hline $1-45$ & -0.8405 & 0.1814 & 0.0545 \\
$46-376$ & -0.5429 & 0.1675 & -0.1017 \\
\hline
\end{tabular}

$R(\theta)=A \tan (B+\theta)+C$.

The fitting of Eq. (7) is a nonlinear optimization problem, for which the least-squares method (LSQ) was used. The task was to minimize the squared difference between data and the model values. The results of fitting Eq. (7) into the quadrant sensor data are shown in Figs. 13 and 14. The fit in Fig. 14 is not as good as in Fig. 13, because in the data of Fig. 14 there exist artifacts caused by as yet unknown reasons. In general the shape of the data in Fig. 14 seems similar to the data in Fig. 13. The values obtained for parameters $A, B$ and $C$ during sol intervals $1-45$ and $46-376$ are presented in Table 3.

After obtaining all the values for Eq. (7) parameters, the fitted tangential model is used to predict the wind directions for new values measured for the ratio of voltages $R$ by the quadrant sensor. The calibration function defined in Eq. (7) can be used to reconstruct the wind directions during the complete VL1 mission, because only the heater element in the quadrant sensor failed. The thermocouples remained operating nominally; therefore, the ratio $R$ of quadrant sensor voltages should obtain values on the same order of magnitude during the whole VL1 mission. The distributions of values, obtained for $R$, during sols 46-377 and 378-2245 are shown in Figs. 15 and 16, respectively. The algorithm for wind reconstruction in stage 2 is similar to the algorithm presented in Algorithm 1 except that the $R$ value is calculated using Eq. (6) instead of the $F$ value.

\section{Validation of the wind reconstruction algorithm}

\subsection{Error analysis of VL1 sols 1-45}

The error analysis of the wind reconstruction algorithm is done by comparing the data produced by the the algorithm with the VL1 SANMET wind data. Because the VL1 wind measurement instruments remained fully functional for the first 45 sols of the VL1 mission, it is assumed that the data for that period are correct and can be used as a reference for the comparison. The behavior of the algorithm was tracked for every reconstructed sol, and data for the error analysis were gathered simultaneously with the reconstruction. The key indicator for the performance of the algorithm is the absolute difference between SANMET and reconstructed angles:

$\Delta \theta=\left|\theta_{\mathrm{SANMET}}-\theta_{\mathrm{Algo}}\right|$

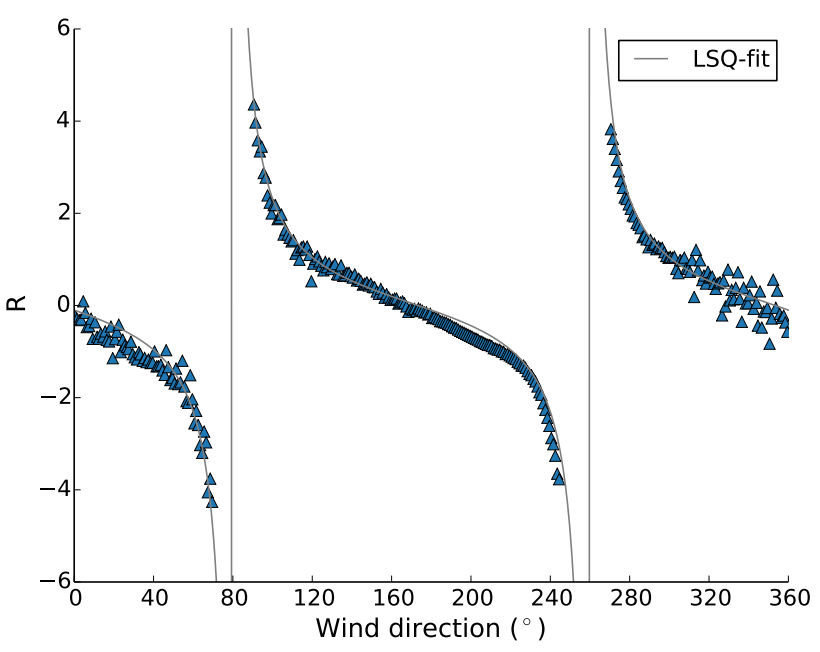

Figure 13. Calibration function $R$ during sols $1-45$.

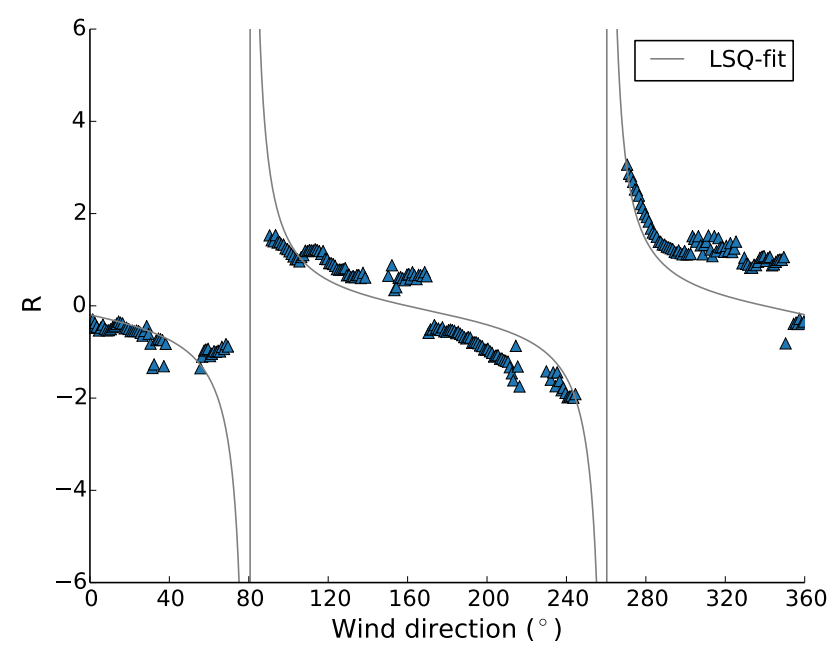

Figure 14. Calibration function $R$ during sols 46-377.

The angle difference of Eq. (8) was calculated for every measurement sample of the reconstructed sols; for each sol reconstructed, a mean value for the angle difference and standard deviation was calculated. The mean of the angle difference and the standard deviation during sols 1-45 are shown in Figs. 17 and 18. The mean value of the mean of the angle differences is $12.8^{\circ}$, and the mean value of the standard deviations is $11.5^{\circ}$. By visually inspecting Fig. 17, one sees that the means of the angle difference vary mostly in the range from 5 to $15^{\circ}$, which is a quite reasonable variation for determining the correct point of compass for the Martian tides.

The reconstruction of wind direction is very accurate during the first 45 sols of the VL1 mission. The reconstructed wind speed shows reasonable behavior as the wind speed begins to increase after sunrise around the seventh hour of LLT. The standard deviations in the reconstructed wind speed are much larger than in the SANMET wind speeds. In general, 


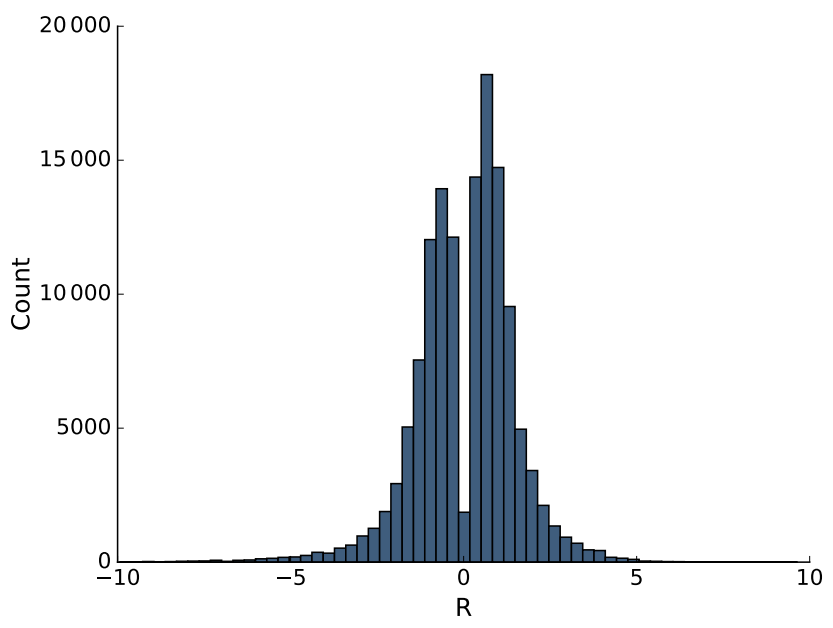

Figure 15. The distribution of $R$ values during sols 46-377.

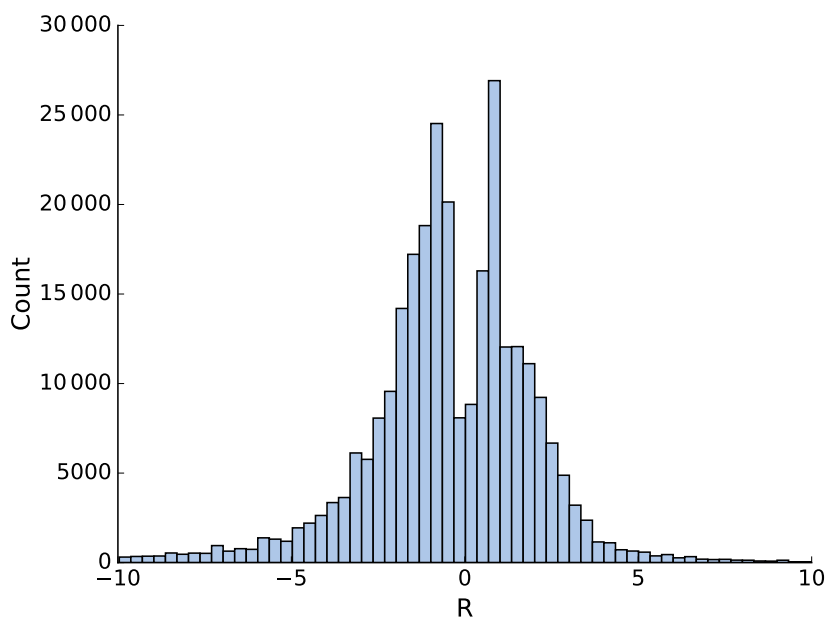

Figure 16. The distribution of $R$ values during sols 378-2245.

the hourly mean of the reconstructed wind speed is greater than the SANMET-determined mean value. However, the SANMET-determined mean value for wind speed is within the error limit of the reconstructed wind speed in all hours except the 20th and 21st. In Fig. 19 the hourly means of SANMET determined wind directions and speeds and the reconstructed wind directions and speeds are shown during the first 45 sols of VL1 mission. The error bars in Fig. 19 are the standard deviations of the measurements done during a specific hour. In addition to the hourly mean wind direction and speed, a point-by-point comparison between the SANMET and reconstructed data are shown in Figs. 20 and 21. Figure 20 presents the comparison between wind direction and speed of SANMET and reconstruction from the forenoon of sol 3. The mean absolute difference between the SANMET and reconstructed wind direction was $9.1^{\circ}$ during the 6 min time period. The reconstructed wind speeds have a high-speed bias caused by the wind direction being almost

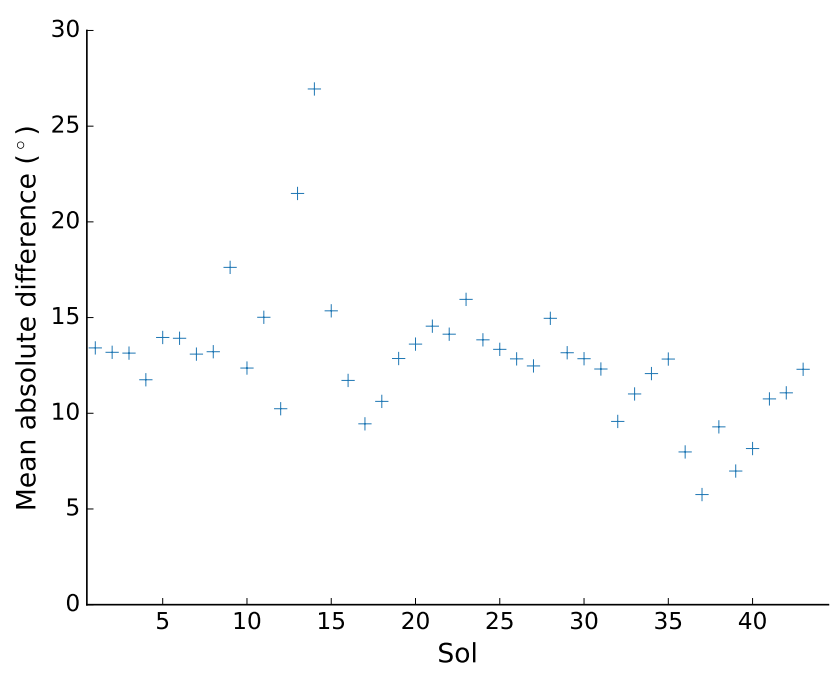

Figure 17. The diurnal mean angle difference.

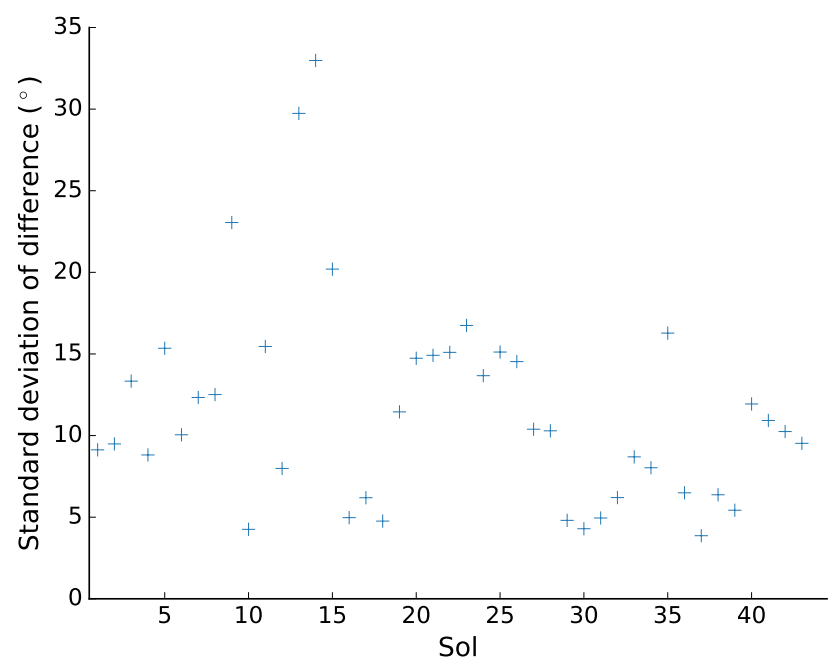

Figure 18. The diurnal standard deviation of the angle difference.

parallel to the WS1. Figure 21 displays SANMET and reconstructed wind directions and speeds during a $1 \mathrm{~h}$ period at the midday of sol 41 . During the $1 \mathrm{~h}$ period, the mean absolute difference between SANMET and reconstructed wind directions was $12.2^{\circ}$.

The method by which a particular measurement sample was determined was also recorded during the reconstruction process. In total, $45 \mathrm{VL} 1$ sols were reconstructed for the analysis. During these sols, 53070 samples were measured. During the reconstruction process 52570 samples $(99.1 \%)$ were determined using the look-up table in Table 2; 500 samples $(0.9 \%)$ were obtained using time continuity. The look-up table was used for the majority of the time to determine the wind direction as there was no threshold value for voltage signals, because the signals were bias-corrected and assumed therefore to be correct. The situations in which time continu- 


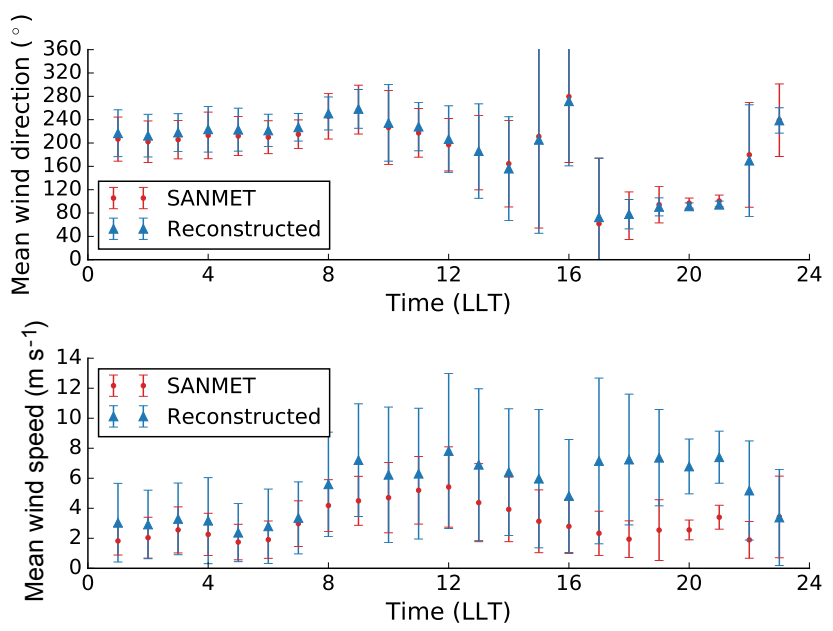

Figure 19. The hourly mean wind direction and speed from SANMET and reconstruction during sols $1-45\left(L_{s}=97-118^{\circ}\right)$.
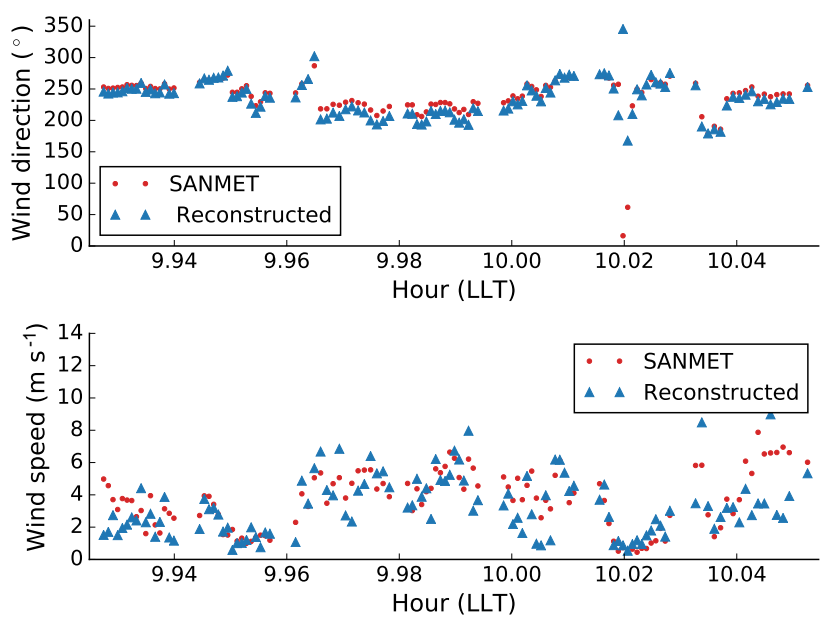

Figure 20. Wind direction and speed during a 6 min time period from the forenoon of $\operatorname{sol} 3\left(L_{S}=98^{\circ}\right)$.

ity was applied in the reconstruction process occurred during nighttime hours (00:00-06:00), when the Sun's radiation was not strong enough to heat the quadrant sensor. In Figs. 22 and 23 , the histograms of the mean difference of reconstructed and SANMET angles as well as the standard deviation of the angle difference are presented.

\subsection{Slope winds of the VL1 area}

Another validation method for the reconstruction algorithm is based on the physical fact that there should exist slope winds in the reconstructed wind data. The VL1 landed on a slope rising to the south and west. In this case the slope winds in the VL1 area will form when the nocturnally cooled dense air is accelerated down the slope by gravity. Therefore, in nocturnal hours, the direction of wind should be in the interval $180-270^{\circ}$, in which case the wind is from the top part
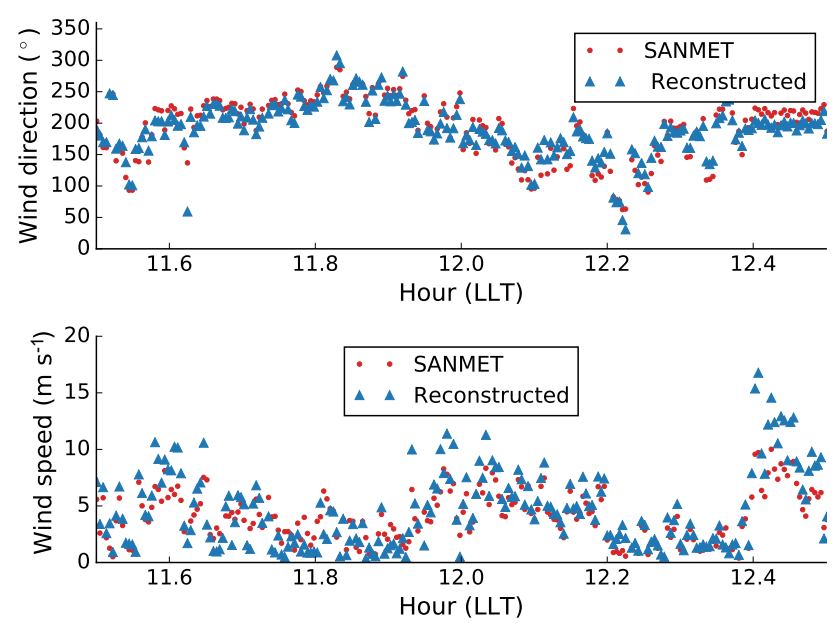

Figure 21. Wind direction and speed during $1 \mathrm{~h}$ at the midday of sol $41\left(L_{S}=116^{\circ}\right)$.

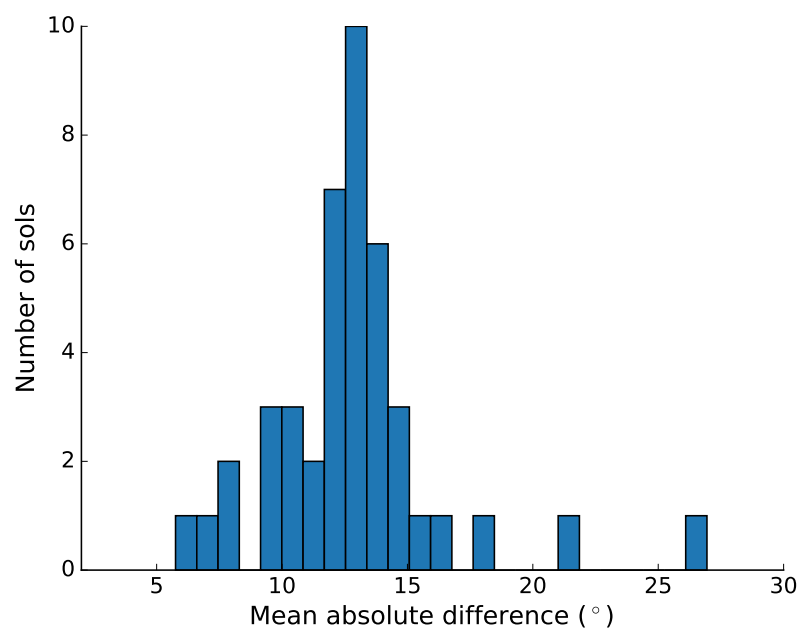

Figure 22. Mean absolute difference of the angles during sols 1-45.

of the slope. Soon after sunrise, the direction of wind should change, so that the daytime winds are anabatic. The anabatic winds are accelerated upslope by sun-heated warm slopes. The daytime wind directions should regularly be in the interval 0-90 ${ }^{\circ}$. According to Figs. 19, 26 and 27, the wind direction data match the physical slope wind directions reasonably well, especially during nighttime and late afternoons. The slope winds are typically observed in Martian summer as the conditions for observing the slope winds are optimal. The conditions for observing the slope wind are a terrain sloping over a large area; strong diurnal temperature variation; and weak ambient winds, which are typical on Mars in summer (Savijärvi and Siili, 1993).

The statistical distribution of wind directions was examined during sols 1-45; the distributions are presented in Fig. 24 with $10^{\circ}$ bin sizes. The bin with the largest count for SANMET wind directions is $200-210^{\circ}$, while for the recon- 


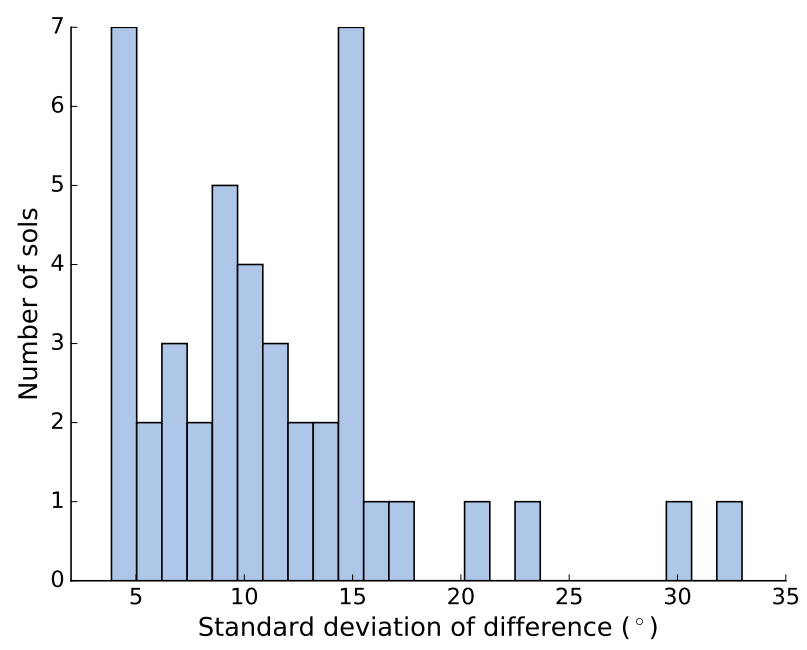

Figure 23. Standard deviation of the angle difference during sols $1-45$.

structed wind directions it is $220-230^{\circ}$. Taking into account that the mean difference between SANMET wind directions and reconstructed directions was $12.8^{\circ}$ during sols $1-45$, the locations of the peak maximums are consistent with each other. Inspecting the left-side tail of both distributions, one sees that there exists a small local maximum approximately between 80 and $100^{\circ}$. The small local maximum is likely caused by the daytime anabatic winds, which flow upslope.

\section{Reconstructed sols of VL1}

The algorithm was used to reconstruct all available VL1 sols, and the results from the reconstruction are presented in this section. The statistical distribution of wind directions is shown in a histogram in Fig. 25 to allow studying the existence of slope winds in the VL1 area. In the figure the wind directions are divided into $10^{\circ}$ sized bins. The distribution of the wind directions determined by SANMET is shown in Fig. 25 as a comparison with the reconstructed wind directions. The SANMET method for determining the wind direction is not reliable after sol 45 , because it does not take into account the decay of the quadrant sensor heater element or, likewise, the failure of one of the wind sensors.

Two clear peaks are visible in Fig. 25 in the reconstructed wind directions. One peak is located between 80 and $120^{\circ}$, and the other is in interval $240-300^{\circ}$. The peak in the angle range $80-120^{\circ}$ is much sharper than the peak in the range $240-300^{\circ}$. The peak in the range $240-300^{\circ}$ corresponds to the nocturnal wind, which is directed downslope, and the peak in the range $80-120^{\circ}$ is the daytime anabatic upslope wind.

To illustrate the reconstructed wind measurements, sols 15 and 1413 of the VL1 mission are presented in Figs. 26 and 27. These sols were selected because the first sol is from
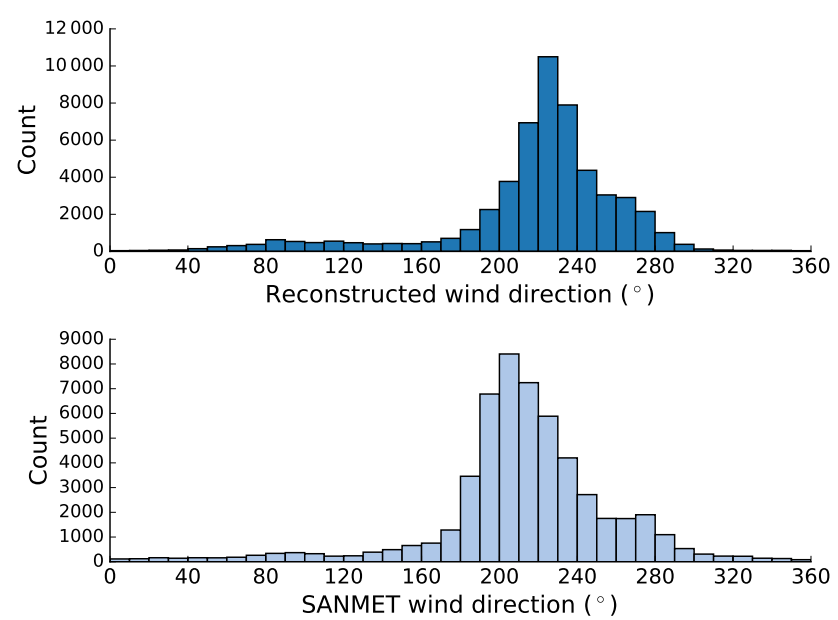

Figure 24. Statistical distributions of wind directions during VL1 sols $1-45$.
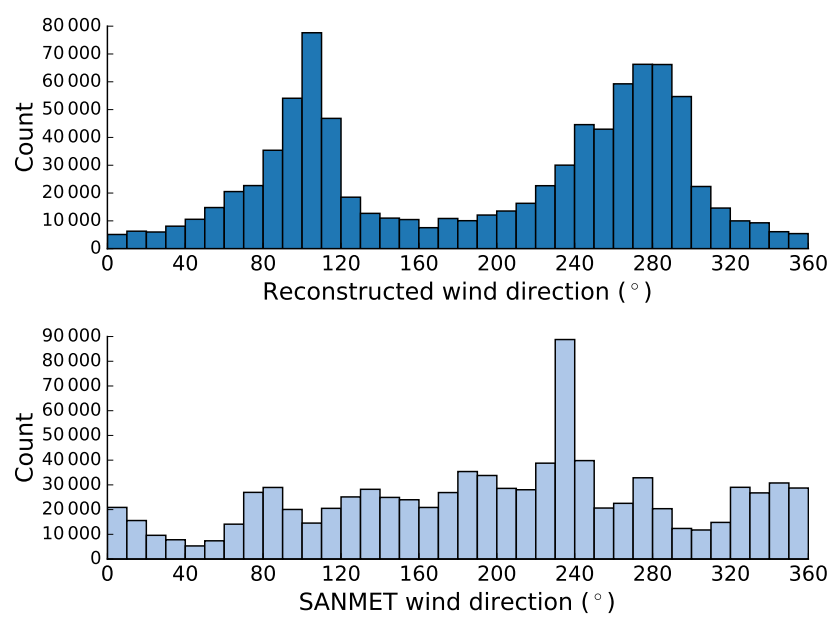

Figure 25. Comparison between SANMET and reconstructed wind direction during the complete VL1 mission.

the time when the VL1 wind measurement instruments were intact, and the second sol is from the time when the wind measurement instruments were partially decayed. The quadrant sensor of VL1 and both of the wind sensors were intact during sol 15. During sol 1413, both the quadrant sensor heater and one of the hot-film wind sensors had already failed. The season is the same in sols 15 and 1413; thus the sols should exhibit similar diurnal behavior.

The hourly mean of wind directions and speeds for both sols are shown in Figs. 26 and 27. Both of the inspected sols occur during the Martian summer, sol 15 being the first year's summer and sol 1413 the third year's summer of the VL1 mission. Thus the conditions for observing slope winds should be favorable. The wind direction is in the range 220$280^{\circ}$ during the nocturnal hours of Figs. 26 and 27, which roughly corresponds to the wind flowing from the upper part 


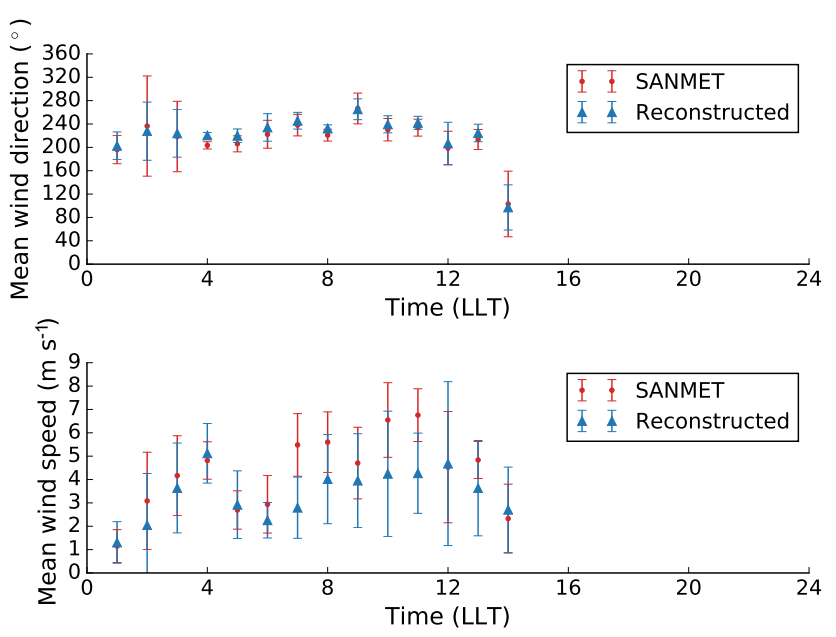

Figure 26. Comparison of SANMET and reconstructed hourly wind directions and speeds for sol 15.

of the slope. After sunrise the wind rotates about $180^{\circ}$ and begins to flow from the lower part of the slope.

\section{Summary and discussion}

The article focused on developing an algorithm to reconstruct the wind measurements during the complete VL1 mission. VL1 performed wind direction and speed measurements on the surface of Mars for 2245 sols; thus the data set produced by the wind reconstruction is significant in its size.

The wind measurement system of VL1 consisted of two orthogonal hot-film wind sensors and a quadrant sensor for solving the ambiguity in wind direction. The quadrant sensor failed during sol 45, and one of the wind sensors broke down during sol 378; thus only one of these three instruments remained fully intact for the VL1 mission. However, with the algorithm described in this report, it was possible to reconstruct the wind measurements with a reasonable accuracy.

The wind reconstruction was completed in two stages. In the first stage the quadrant sensor signals from sols 1-45 were used to calibrate Nusselt numbers of the hot-film wind sensors. The wind sensors were then used to estimate wind directions during sols 46-377. With the estimated wind directions the quadrant sensor was recalibrated and was then used to reconstruct all the wind directions from the VL1 mission. The reconstruction of wind speed was also possible with one wind velocity component and the wind direction. The results from the reconstruction of the wind speeds are not always very reliable, because the reconstruction of wind speed required knowledge of both the wind direction and the wind velocity component from the nominally working wind sensor. The wind directions contain variable amounts of error between different sols. Therefore, the reconstruction quality of the wind speed is weaker for sols with more error in the wind direction.

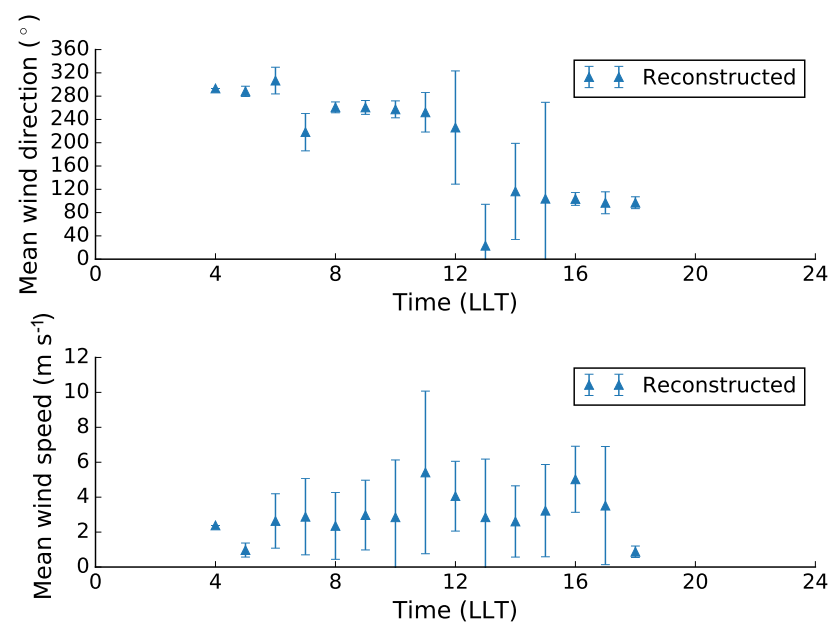

Figure 27. The reconstructed hourly wind directions and speeds for sol 1413.

The developed algorithm for wind reconstruction shows the presence of slope winds in the VL1 area. The accuracy of the algorithm compared to the data measured by fully functional VL1 wind measurement instruments is reasonable. On average the mean of the absolute difference between wind direction was determined to be $12.8^{\circ}$.

The new wind reconstruction algorithm developed in this article extends the amount of available sols of VL1 from 350 to 2245 sols. The reconstruction of wind measurement data enables the study of both short-term phenomena, such as daily variations in wind conditions or dust devils, and longterm phenomena, such as the seasonal variations in Martian tides.

Data availability. The data are not publicly available as they are currently in an immature state but will be made completely available later on, likely through the PDS. At present the data are available upon request; please bear in mind that the data may be subject to changes.

Competing interests. The authors declare that they have no conflict of interest.

Acknowledgements. The authors are thankful for the Finnish Academy grant no. 131723.

Edited by: Valery Korepanov

Reviewed by: Jim Murphy and one anonymous referee 


\section{References}

Buehler, G. D.: SD-37P0011A Viking'75 Project Program Description Document for the Meteorology Analysis Program (SANMET), Project documentation, National Aeronautics and Space Administration (NASA), 1974.

Chamberlain, T. E., Cole, H. L., Dutton, R. G., Greene, G. C., and Tillman, J. E.: Atmospheric Measurements on Mars: the Viking Meteorology Experiment, B. Am. Meteorol. Soc., 57, 1094-1104, https://doi.org/10.1175/15200477(1976)057<1094:AMOMTV>2.0.CO;2, 1976.

Davey, R., Chamberlain, T., and Harnett, L.: Sensor Design Analysis Report, TRW Systems Group, 1973.

Golombek, M. P., Anderson, R. C., Barnes, J. R., Bell III, J. R., Bridges, N. T., Britt, D. T., Brückner, J., Cook, R. A., Crisp, J. A., Economou, T., Folkner, W. M., Greely, R., Haberle, R. M., Hargraves, R. B., Harris, J. A., Haldemann, A. F. C., Herkenhoff, K. E., Hviid, S. F., Jaumann, R., Johnson, J. R., Kallemeyn, P. H., Keller, H. U., Kirk, R. L., Knudsen, J. M., Larsen, S., Lemmon, M. T., Madsen, M. B., Magalhães, J. A., Maki, J. N., Malin, M. C., Manning, R. M., Matijevic, J., McSween, H. Y., Moore, H. J., Murchie, S. L., Murphy, J. R., Parker, T. J., Reider, R., Rivellini, T. P., Schofield, J. T., Seiff, A., Singer, R. B., Smith, P. H., Soderblom, L. A., Spencer, D. A., Stoker, C. R., Sullivan, R., Thomas, N., Thurman, S. W., Tomasko, M. G., Vaughn, R. M., Wänke, H., and Wilson, G. R.: Overview of the Mars Pathfinder Mission: Launch Through Landing, Surface Operations, Data Sheets, and Science Results, 1999.

Hess, S., Henry, R., Leovy, C. B., Ryan, J., and Tillman, J. E.: Meteorological Results from the Surface of Mars: Viking 1 and 2, J. Geophys. Res., 82, 4559-4574, 1977.

Holstein-Rathlou, C., Gunnlaugsson, H. P., Merrison, J. P., Bean, K. M., Cantor, B. A., Davis, J. A., Davy, R., Drake, N. B., Ellehoj, M. D., Goetz, W., Hviid, S. F., Lange, C. F., Larsen, S. E., Lemmon, M. T., Madsen, M. B., Malin, M., Moores, J. E., Nürnberg, P., Smith, P., Tamppari, L. K., and Taylor, P. A.: Winds at the Phoenix landing site, J. Geophys. Res.-Planets, 115, e00E18, https://doi.org/10.1029/2009JE003411, 2010.
Kemppinen, O., Tillman, J., Schmidt, W., and Harri, A.-M.: New Analysis Software for Viking Lander Meteorological Data, Geoscientific Intrumentation Methods and Data Systems, 2013.

Murphy, J. R., Leovy, C. B., and Tillman, J. E.: Observations of Martian Surface Winds at the Viking Lander 1 Site, J. Geophys. Res., 95, 14555-14576, 1990.

Newman, C. E., Gómez-Elvira, J., Marin, M., Navarro, S., Torres, J., Richardson, M. I., Battalio, J. M., Guzewich, S. D., Sullivan, R., de la Torre, M., Vasavada, A. R., and Bridges, N. T.: Winds measured by the Rover Environmental Monitoring Station (REMS) during the Mars Science Laboratory (MSL) rover's Bagnold Dunes Campaign and comparison with numerical modeling using MarsWRF, Icarus, 291, 203-231, https://doi.org/10.1016/j.icarus.2016.12.016, 2016.

Savijärvi, H. and Siili, T.: The Martian Slope Winds and the Nocturnal PBL Jet, J. Atmos. Sci., $\quad 50, \quad 77-88, \quad$ https://doi.org/10.1175/15200469(1993)050<0077:TMSWAT>2.0.CO;2, 1993.

Schofield, J., Barnes, J. R., Crisp, D., Haberle, R. M., Larsen, S., Magalhaes, J., Murphy, J. R., Seiff, A., and Wilson, G.: The Mars Pathfinder Atmospheric Structure Investigation/Meteorology (ASI/MET) Experiment, Science, 278, 1752 1758, 1997.

Soffen, G. A.: The Viking Project, J. Geophys. Res., 82, 3959-3970, 1977.

Sutton, J. L., Leovy, C. B., and Tillman, J. E.: Diurnal Variations of the Martian Surface Layer Meteorological Parameters During the First 45 Sols at Two Viking Lander Sites, J. Atmos. Sci., 35, 2346-2355, https://doi.org/10.1175/15200469(1978)035<2346:DVOTMS>2.0.CO;2, 1978 . 